\title{
Band Gap Computation of 1-Dimentional Silicon Photonic Crystal for High Contrast Grating Application
}

\author{
Gagandeep Kaur \\ M.Tech \\ Department of Electronics and \\ Communication Engineering, \\ Punjabi University, Patiala
}

\author{
Amandeep Singh Sappal \\ Assistant Professor \\ Department of Electronics and \\ Communication Engineering, \\ Punjabi University, Patiala
}

\author{
Harjinder Singh \\ Assistant Professor \\ Department of Electronics and \\ Communication Engineering, \\ Punjabi University, Patiala
}

\begin{abstract}
One Dimensional Photonic Crystal (PHc) is manageable and uncomplicated type of PHc, It refers to the fact that the dielectric is only periodic in one direction. The evaluation of photonic structure by numerical and a simulation method is hugely significant. At optical frequencies the optical thickness inside one dimensional $\mathrm{PHc}$ changes periodically. They have the attributes to robust effect the propagation of light waves at these optical frequencies. In the instant work, band-gap has calculated for one dimension PHc. The Finite Difference Time Domain (FDTD) and plane wave expansion (PWE) Methods are extensively used for band gap calculation of PHc's. FDTD Method has more flexibility and simplicity and can be explored easily for computation of the field distribution inside the photonic structure than PWE method so we have used FDTD Method for one dimensional PHc's calculation.
\end{abstract}

\section{General Terms}

FDTD Algorithm using High Contrast Grating Application.

\section{Keywords}

Band gap, FDTD, PWE, Photonic Band-gap material, One Dimensional Photonic Crystal, High Contrast Grating.

\section{INTRODUCTION}

A Key Technology of $21^{\text {st }}$ century is nanotechnology. It explores extremely small structures of the size of a few nanometers up to several 100 nano-meters. Thus, the wavelength of light is often greater then these small structures. The PHc are significant class of physical structure studied in nanotechnology. [1]

The need for PHc is usually darken in public eye by the surprising convenience of fiber optic wires. The accurate fiber optic wires, as well as plastic wires are proficient to curve at small extent before either signal loss or cable breakage set in. Furthermore, fiber optic cables receive impressive signal loss over long distance, the signal must be reinforce by the repeaters along the wire or by transmitter in order to be observable by the destination. PHc overcome this problem, go through very little signal loss related to the finest fiber optic wires that we have used today [2]. PHc are generally 'honeycomb' structure made of silicon. PHc can be divided into three broad categories, namely one dimensional (1D), 2 dimensional (2D), and three dimensional (3D) structures. Illustration of 1D, 2D and 3D PHc are shown in figure (1). [4] In one dimension $\mathrm{PHc}$, the periodic modulation of the permittivity occurs in one direction only, while two directions structure is stable. The one dimensional $\mathrm{PHc}$ are made of layers of material arranged together with nanoscopic space between them to serve as channel for light to pass through. The material serves as a shield to keep the light from escaping.

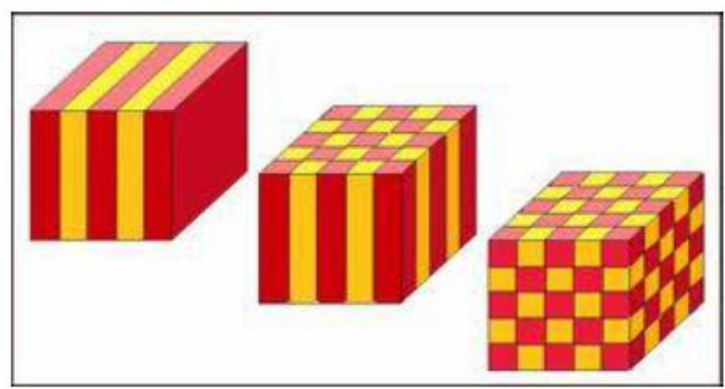

Figure 1: Examples of (a) 1D Photonic crystal (b) 2D Photonic crystal (c) 3D Photonic crystal

Example of such a one dimensional PHc, It can be given the Bragg grating, which is most extensively used as a distributed reflector in cavity (which is vertical in nature) surface emitting lasers. 1D crystal only allows the light to propagate in a one straight line with no changes of direction. However 2D and 3D PHc's have extremely large potential in upcoming technologies.2D PHc can have moderately large different types of groups, because its periodic modulation of the permittivity occurred in two directions while in the third direction. The medium is stable. A good example of $2 \mathrm{D} \mathrm{PHc}$ is porous silicon, which are arranged the pores periodically. Which shown by the silicon substrate with etched holes. 3D PHc has permittivity modulation along all three directions.

The most known naturally formed 3D PHc is valuable stone opal. These types of stones known by its unconventional optical properties. [4]

In 1990, K.M. Ho, C.T. Chan and C.M. Soukoulis [5] obtained the band structures of the Photonic crystal with Face Centered Cubic (FCC) lattices (opal structure) which consist of dielectric spheres with high refractive index in air. Band gap structures were computed. The Investigations show the existence of at least one Eigen-state exists, so that's way no complete Photonic band gap appears. These type of causes the optical effects typical for all natural and artificial opals. In the same work, there were also gives some results of band structure computation for the Photonic crystal with diamond lattice structure made of dielectric spheres situated in air, where they found complete Photonic band gap between the second and third bands. 


\section{MATHEMATICAL PRELIMINARIES FOR CALCULATION OF BANDGAP}

To Simulate the Band gap structure of one dimensional PHc, It is indispensable to solve the Eigen problem for the one dimensional Helmholtz equation. [4] The Helmholtz equation for the Electric field is given in (1)

$\frac{\partial^{2}(\mathrm{EZ}(\mathrm{x}))}{\partial \mathrm{x}^{2}}+\frac{\epsilon \mathrm{r}(\mathrm{x}) \mathrm{w}^{2} \mathrm{Ez}(\mathrm{x})}{\mathrm{c}^{2}}=0$

So simulation of Band gap structure is more appropriate to carry out using the equation for Magnetic field component. The equation in Magnetic field component case takes as follows:

$\frac{1}{\epsilon(\mathrm{x})}=\sum \mathrm{G}^{\prime \prime} \in \mathrm{G} x\left(\mathrm{G}^{\prime \prime}\right) \cdot \mathrm{e}^{\left(\mathrm{J} \cdot \mathrm{G}^{\prime \prime} \cdot \mathrm{x}\right)}$

The difference of above equation from the equation (1) lies in an operator. Here in equation (2) dielectric function stands inside one of the co-ordinate derivatives, While in equation (1) it is outside all derivatives. The dielectric function inside the operator occurs some tribulations when finding for field distribution inside the structure; such a form of Helmholtz equation is quite useful for band gap structure computation. [6]

In order to solve out the Eigen value equation (2) some methods should be used which applied in the periodicity of the permittivity distribution. Some time, we learn that the Eigen function of an infinite periodic structure will also be infinite and periodic. That is way the Bloch theorem should be used for describing the Eigen function of PHc. In the Bloch Theorem, such function has the following form [7]

$H(x)=h_{k, n}(x) e^{(j . k . x)}$

Where:-

$\mathrm{h}_{\mathrm{k}, \mathrm{n}}(\mathrm{x})=$ periodic function with periodicity of lattice

$\mathrm{k}=\quad$ wave vector

$\mathrm{n}=\quad$ state number

As we can't continue with the simulation of an infinite function inside equation, so we broaden the function (2) to Fourier series by reciprocal lattice vectors.

$H(x)=\sum_{G} h_{k, n}(G) e^{(j \cdot(k+G) \cdot x)}$

Where:-

$\mathrm{h}_{\mathrm{k}, \mathrm{n}}(\mathrm{G})$ - formerly defined periodic function in describing of wave vector

Now enlarge the Fourier series not permittivity but the inverted dielectric function as it written in equation (2) when it enlarges, after that we substitute all infinite function in equation (2) is:

$\sum \frac{\partial\left(\mathrm{x}\left(\mathrm{G}^{\prime \prime}\right)\right.}{\partial \mathrm{x}} \cdot \mathrm{e}^{\left(\mathrm{J} \cdot \mathrm{G}^{\prime \prime} \cdot \mathrm{x}\right)} \frac{\partial \sum \mathrm{h}_{\mathrm{k}, \mathrm{n}}(\mathrm{G}) \mathrm{e}^{(\mathrm{j} \cdot(\mathrm{k}+\mathrm{G}) \cdot \mathrm{x})}}{\partial \mathrm{x}}$

$+\frac{\mathrm{w} 2}{\mathrm{c} 2} \sum \mathrm{Gh}_{\mathrm{k}, \mathrm{n}}(\mathrm{G}) \mathrm{e}^{(\mathrm{j} \cdot(\mathrm{k}+\mathrm{G}) \cdot \mathrm{x})}=0$

Now Substitute $\mathrm{G}=\mathrm{G}^{\prime}+\mathrm{G}^{\prime}$

So $G^{\prime \prime}=(G-G ')$ form [8], [9]

$$
\begin{aligned}
& \sum_{G} \sum_{G^{\prime}} \frac{\partial\left(\mathrm{x}\left(\mathrm{G}-\mathrm{G}^{\prime}\right)\right)}{\partial \mathrm{x}} \cdot \mathrm{e}^{\left(\mathrm{j} \cdot\left(\mathrm{G}-\mathrm{G}^{\prime}\right) \cdot \mathrm{x}\right)} \cdot \mathrm{h}_{\mathrm{k}, \mathrm{n}}\left(\mathrm{G}^{\prime}\right) \frac{\partial \mathrm{e}^{(\mathrm{j} \cdot(\mathrm{k}+\mathrm{G}) \cdot \mathrm{x})}}{\partial \mathrm{x}} \\
& +\frac{\mathrm{w}^{2}}{\mathrm{c}^{2}} \sum \mathrm{G}^{\prime} \mathrm{h}_{\mathrm{k}, \mathrm{n}}(\mathrm{G}) \mathrm{e}^{(\mathrm{j} \cdot(\mathrm{k}+\mathrm{G}) \cdot \mathrm{x})}=0
\end{aligned}
$$

Then taking Derivative and summed up the exponents and we get

$$
\begin{aligned}
& \sum_{G} \sum_{G^{\prime}} \mathrm{x}\left(G-G^{\prime}\right) \cdot \mathrm{h}_{\mathrm{k}, \mathrm{n}}\left(G^{\prime}\right) \cdot \mathrm{e}^{\left(\mathrm{j} \cdot\left(\mathrm{G}-\mathrm{G}^{\prime \prime}\right) \cdot \mathrm{x}\right)} \mathrm{X}(\mathrm{j} \cdot(\mathrm{K}+\mathrm{G}) \cdot \mathrm{j} \cdot(\mathrm{k}+\mathrm{G}) \\
& +\frac{\mathrm{w}^{2}}{\mathrm{c}^{2}} \sum \mathrm{G}^{\prime} \mathrm{h}_{\mathrm{k}, \mathrm{n}}(\mathrm{G}) \mathrm{e}^{(\mathrm{j} \cdot(\mathrm{k}+\mathrm{G}) \cdot \mathrm{x})}=0
\end{aligned}
$$

The estimation on the basis $\mathrm{e}^{(\mathrm{j} \cdot(\mathrm{k}+\mathrm{G}) \cdot \mathrm{x})}$ gives the equation:

$$
\begin{aligned}
& -\sum_{\mathrm{G}^{\prime}} \mathrm{x}^{\prime}\left(\mathrm{G}^{\left.-\mathrm{G}^{\prime}\right)}\left((\mathrm{k}+\mathrm{G}) \cdot\left(\mathrm{k}+\mathrm{G}^{\prime}\right)\right) \cdot \mathrm{h}_{\mathrm{k}, \mathrm{n}}\left(\mathrm{G}^{\prime}\right)\right. \\
& +\frac{\mathrm{w}^{2}}{\mathrm{c}^{2}} \mathrm{~h}_{\mathrm{k}, \mathrm{n}(\mathrm{G})}=0
\end{aligned}
$$

This is called as "Master Equation" for one dimensional PHc. where $x(G$ ") is Fourier expansion coefficient of the inverted dielectric function.

The group of wave vectors the simulation is carried out for should be elected taking into explanation the specificity of the difficulties. For a few time, the verification of Eigen state of the periodic structure with unsmoothed (change of one of PHc elements thickness or permittivity which causes the light localization) one value of wave vector is essential, while simulation of pure periodic structure require a group of wave vector taken in some significant points within the Brillouin zone. To solve out a more difficult characteristics such as photonic density of state it is essential to solve out the Eigen state in all points of brillouin zone. This matrix is one with elements which satisfy the following condition. [4]

\section{FDTD ALGORITHM FOR BANDGAP CALCULATION}

One of the best ways to get control the difficulty of PWE is to apply the FDTD method. In opposition to PWE technique, The FDTD allocate possibilities of the refractive index changes during the mathematically calculation procedure. [10]

Which gives permission to take into account nonlinearity and losses, when solve out the band structure. H. Yu. D. Yang in 1966 proposed FDTD algorithm. The most efficient used statistical method in meta-materials study is FDTD method. It is used for the direct solve out the Maxwell's equation, FDTD method provide an easy way to model difficult periodic structures. The formulation of Yang's FDTD algorithm, the yang algorithm exist at same time deal with both magnetic and electric field in time and space using the coupled from of Maxwell's curl equations.

In general, PBG simulation using FDTD should be carried out as follows:-

(1) Calculate the computation area.

(2) Configure the periodic boundary conditions.

(3) Explanation of the radiation excitation function. The radiation spectrum should be broad sufficient to cover fully explore the frequency range.

(4) Takeaway the spectral investigation of the Time dependent response of the construction on the probe 
pulse by finding all of local maxima and plotting them over frequency axis.

(5) Replicate the steps from 2 to 4 at different values of the phase shift in periodic boundary conditions comparable to all selected points within the PHc Brillouin zone the Band Structure is solve-out for the program initialization is execute inside the $1^{\text {st }}$ block.

In this program, firstly, we have created the structure geometry, exceptionally, the thickness and period of layers, as well as permittivity scatter inside the each layer of PHc. Here the design is very manageable so we are setting up only the fraction of period alternatively of entire thickness of the layer. The second, third and fourth blocks are inside the loop, which are scans above the wave-vectors. The second block created the FDTD Method for the one dimensional case. The spatial response with in determine points obtained at the block output. The spatial response examination is workout in third block. Here, the FFT is put into spatial response, which gives its spectrum, after that, the spectrum is scanned and found the local maxima.

Each maximum values correlate with Eigen state of $\mathrm{PHc}$ at given value of wave-vector. Eigen State achieves for particular value of Wave-vector are then mark out one by one in the Figure. Since the Eigen state is investigates for different points or values of wave-vector, then we finally get the band gap structure. The final results of band gap structure simulation is shown in figure (2).As in figure seen, some further solutions which are appear in figure which is drop-out from the general set. It is called unauthentic solutions. Such solution appears due to space discretization and because of Finite Solution Time Intervals. Such solution may easily be removed even manually.

Table 1. Parameters Used For Band-Gap Material (Silicon) of One Dimensional Photonic Crystal

\begin{tabular}{|l|c|}
\hline \multicolumn{1}{|c|}{ Parameters } & Values \\
\hline Lattice Constant & $0.543 \mathrm{~nm}$ \\
\hline Refractive Index & 1.46 \\
\hline Accuracy & 48 \\
\hline Thickness of Layers & L1 $=0.3$ \\
\hline Light Speed in Vacuum & $3 \mathrm{e}=0.7$ \\
\hline Method & FDTD Algorithm \\
\hline
\end{tabular}

Table 2. Band gap of one dimensional photonic crystal

\begin{tabular}{|l|l|}
\hline \multicolumn{1}{|c|}{ S.No. } & $\begin{array}{c}\text { Approximation Band Gap Between } \\
\text { Different Frequencies Ranges from Fig.2 }\end{array}$ \\
\hline 1. & 0.3 to $0.6 \mathrm{GHz}$ \\
\hline 2. & 0.8 to $1.2 \mathrm{GHz}$ \\
\hline 3. & 1.49 to $1.75 \mathrm{GHz}$ \\
\hline 4. & 2.1 to $2.6 \mathrm{GHz}$ \\
\hline 5. & 2.9 to $3.1 \mathrm{GHz} \quad \ldots$ so on \\
\hline
\end{tabular}

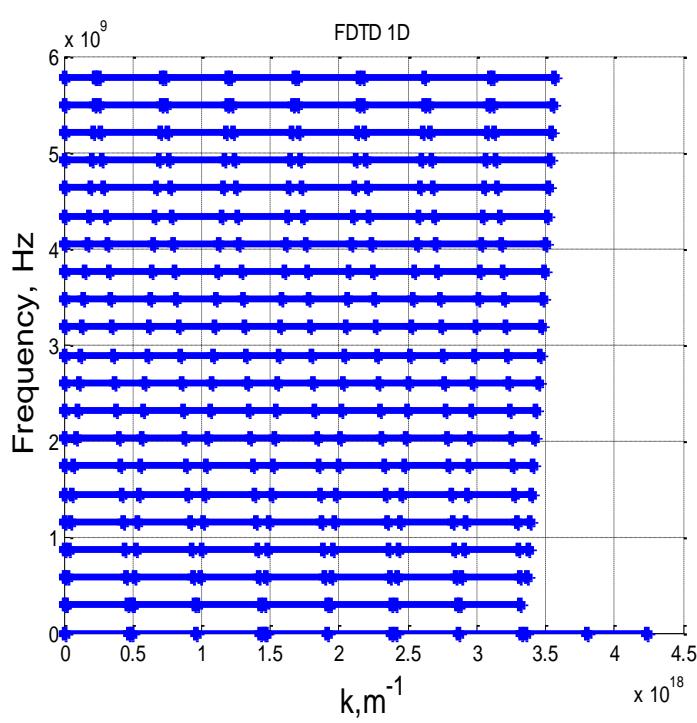

Fig.2. Simulation Results for One Dimension Photonic Band-Gaps in between the frequency "wa/2 $\pi \mathrm{c}$ " is plotted versus the wave vector " $k$ " using FDTD Method.

\section{CONCLUSION}

The Band-gap from Fig (2) I have concluded the band-gap of silicon is uniform and hence it can be used designing nanooptical devices like High Contrast Grating.

The light signals that are passing through a PHc affected from obstruction inside the crystal and depend upon the frequency of Incoming light signal, this problem can either be critical or beneficial. In other cases the light signal is not able to travel inside the PHc. The range of optical frequencies at which this event created is known as Photonic band gap. Thus light signal with frequency inside PBG are fully reflected by PHc.

In future the scope of the band gap for 2Dimensional, 3Dimensional Photonic crystals can be calculated by using FDTD algorithm or the PWE method also for the design of High Contrast Grating can be customized so as to achieve very high reflectivity $(99.9 \%)$. 


\section{ACKNOWLEDGMENTS}

Gagandeep Kaur, Author wishes to express her sincere gratitude to Dr. Amandeep Singh Sappal and Mr. Harjinder Singh, University College of Engineering, Punjabi University Patiala, for guiding her throughout the current research work.

\section{REFERENCES}

[1] L. Rayleigh, on the maintenance of vibrations by forces of double frequency, and on the propagation of waves through a medium endowed with a periodic structure. Philosophical Magazine 24, 145- 159 (1887).

[2] V.P. Bykov, Spontaneous emission in a periodic structure. J. Exp. Theor. Phys. 35, 269 (1972).

[3] E. Yablonovitch, Inhibited spontaneous emission in solid-state physics and electronics. Phys. Rev. Lett. 58, 2059-2062 (1987).

[4] Swarnjeet Kaur, Deepak Saini, Amandeep Sappal, Band Gap Simulations of 1-Dimensional Photonic Crystal, Ijarcsee (2012)

[5] K.M. Ho, C.T. Chan, C.M. Soukoulis, Existence of a photonic gap in periodic dielectric structures. Phys. Rev. Lett. 65, 3152-3155 (1990)

[6] K. Sakoda, Optical Properties of Photonic Crystals (First Edition). (Springer, Heidelberg, (2001)

[7] J.-M. Lourtioz, H. Benisty, V. Berger, et al., Photonic Crystals, Towards Nanoscale Photonic Devices. (Springer, Heidelberg, (2005)

[8] K. Yitzhak, An introduction to Harmonic Analysis, 2nd corrected ed. (Dover, NY, 1976)
[9] A. Taflove, S.C. Hagness, Computational Electrodynamics: The Finite- Difference Time-Domain Method, 3rd edn. (Artech House, Norwood, MA, 2005)

[10] H. Yu D. Yang, Finite difference analysis of 2-D photonic crystals. IEEE Trans. microwave Theory Tech. 44(12), 2688-2695 (1996)

\section{AUTHOR'S PROFILE}

Gagandeep Kaur is currently pursuing M.Tech (Final Year) in Department of Electronics and Communication Engineering at University College of Engineering, Punjabi University, Patiala. She received the B.Tech degree in University College of Engineering at Punjabi University, Patiala, India, in 2012. Her currently research interests in Band gap of One Dimensional Photonic crystal in optical communication.

Amandeep Singh Sappal is currently Assistant Professor at University College of Engineering, Punjabi University Patiala, India. He has completed his M.Tech from Punjab Technical University, Jalandhar, India and B.Tech from Punjab University, Chandigarh, India. Mr. Sappal has 13 years of academic experience. He has authored 10 research papers in reputed International journals and more than 15 research papers in National and International conferences. His areas of interests are Optical and Wireless communication, embedded systems and VLSI Design using DSP Techniques.

Harjinder Singh is currently Assistant Professor in University College of Engineering, Punjabi University Patiala, India. He has completed his B.Tech and M.Tech from Guru Nanak Dev Engineering College Ludhiana, Punjab, India. Mr.Harjinder Singh has 14 years of academic experience. His area of interests is Wireless Communication, Signal Processing and Optical Communication. He has to his credit many papers in International journal and national and international Conferences. 